\title{
Assembling a Swarm Navigation System: Communication, Localization, Sensing and Control
}

\author{
Siwei Zhang, Robert Pöhlmann, Emanuel Staudinger and Armin Dammann \\ Institute of Communications and Navigation, German Aerospace Center (DLR), Oberpfaffenhofen, Germany \\ Email: firstname.lastname@DLR.de
}

\begin{abstract}
Autonomous robotic swarms have attracted increasing attention in recent years due to their enormous, principally unlimited, capability expansion from single robot systems. A communication system, as the 'glue' in a swarm, plays an essential role that goes beyond conventional data exchange into localization, sensing, control, etc. Each building block of a swarm can be rooted from specific well-addressed research topic such as communications, signal processing, robotics, control, etc. However, an interdisciplinary view on swarm communication system, specially its impacts on other sub-systems, is still missing. In this paper, we share our experience in the assembling of a radio-based swarm system, from module design to system integration, from theoretical investigation to experimental platform development. With that we aim to shed light on some essential issues when designing a communication system particularly suitable for swarms.
\end{abstract}

\section{INTRODUCTION}

Environmental monitoring, the search for life or resources, particularly in inaccessible areas on earth or in space, is still a challenge today. Exploring huge areas such as the deep sea or planetary surfaces with a single robotic system often appears to be a Sisyphean task or resembles the proverbial 'search for the needle in the haystack'. To maximize the chances of success of exploration missions, it is necessary to explore unknown areas on a much larger scale and in comparably shorter time than it is done today. A major challenge is navigation and control of the exploration system, since such a system usually operates in unknown areas, where no navigation and communication infrastructure is available. Compared to a single robotic system, the application of a swarm of robots for exploration offers numerous advantages:

1) Redundancy \& Reliability: Due to the redundant design, the entire system can tolerate the failure of individual swarm elements.

2) Speed: Due to parallel exploration the time required for exploration is considerably reduced.

3) Coverage: A swarm is connected by a communication mesh network, which enables exploration of wide areas.

4) Navigation accuracy: Cooperative localization methods based on mutual ranging between swarm elements increase navigation accuracy.

5) Large observation aperture and accuracy of exploration: The swarm can be used as a large sensor array, which increases the accuracy of observations and enables new possibilities.

6) Scalability: By controlling the swarm topology, the overall system properties can be adapted to the course of the exploratory mission.

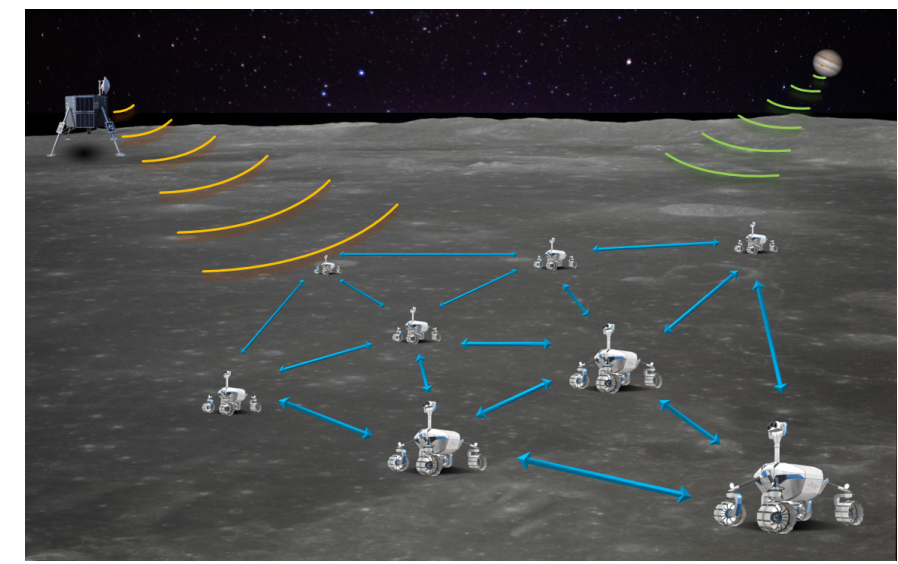

Figure 1: A conceptual swarm lunar mission, where the swarm composes a distributed phased array.

In nature, swarm behavior refers to grouping of numerous biological entities, for example bird flocking or fish schooling [1]. Each entity, or agent, follows simple interaction rules based on the observation of its surrounding [2]. Yet the whole swarm acts as a single organ with emerging global situation awareness and collective behaviors, such as immigrating, foraging or escaping from predators [3]. Autonomous robotic swarms, analogous to biological swarms in nature, are self-organized multi-agent systems composed of a crowd of collaborative artificial entities [4], [5]. Robotic swarm systems attract increasing attention in sensing and exploration applications, e.g. for search and rescue [6], environmental monitoring [7], and extraterrestrial missions [8], [9]. The size of a robotic swarm, referred to either its cardinality or its collective aperture's size, varies depending on the applications. The cardinality, i.e. the number of agents in the swarm, differs from a few dozens in laboratory demonstrations [10] to a few thousands in National Aeronautics and Space Administration (NASA)'s envisioned deep space exploration missions [11]. The collective aperture, i.e. the collective area covered by the swarm, has also a wide range of sizes, from nanometer scale for nano-swarms implanted inside the human body [12], to a few hundred meters for planetary surface swarm sensing and exploration in both terrestrial [13] and extraterrestrial applications [14], and to hundreds of kilometers for orbital applications with a satellite swarm [4].

As an example, a conceptual lunar swarm mission is illustrated in Figure 1. A swarm jointly synchronizes and localizes 
itself with the help of communication links (blue arrows), and drives from the mission base (upper left) to a target area. The formation of the swarm is autonomously optimized, composing a distributed phased array. This array can be used for radio astronomy as a lunar-based radio telescope network, i.e. low frequency array (LOFAR), which senses extraterrestrial radio signals like the Jupiter's radio bursts (colored in green). Once the mission is accomplished, the array's formation can be readjusted for sensing the radio signal from the lander (colored in yellow) in order to return to the mission base.

A robotic swarm system consists of several generic building blocks, which are:

1) Communication: Data exchange among agents is required to enable collaborative behavior. The communication system's physical layer (PHY) and media access control layer (MAC) with its channel access scheme, and protocols must meet requirements on throughput, latency, etc.

2) Localization: Control algorithms for a robotic swarm need to know position as well as orientation of all the agents. The agents' position is also required for sensing scalar fields like temperature or vector fields like wind, water currents and electromagnetic field.

3) Sensing: The fundamental task of exploration is the reconstruction of a spatio-temporal process through parameter estimation based on sensed physical quantities. Expected process change rates, described by partial first order derivatives, proportionally set the requirements on accuracy for self-localization and timing building blocks and determine communication latency.

4) Control: Control algorithms steering the individual agents aim to optimize the exploration progress by, e.g., maximizing the information gain in each control step. In particular it is important to take safety criteria like collision avoidance into account when operating in challenging environments.

5) Timing: All of the building blocks addressed before need knowledge about time among all agents with certain accuracy, depending on the speed of exploration and the dynamics of the physical process which shall be observed.

In this paper we focus on communication, localization, sensing and control out of the building blocks mentioned above and discuss how to assemble a swarm navigation system from these building blocks. Subsequently, we

- introduce the radio signals, which we use for communication, ranging and localization,

- discuss the observation of low-frequency radio signals as an example application for a robotic exploration swarm,

- provide a method for the estimation of a swarm system's state, in particular its agents' positions and orientations,

- address the principle of swarm control, which we use in our swarm system,

- show the evolution of our experimental swarm system platforms and

- present selected results regarding swarm navigation performance.

\section{Radio Signals ExPloited By A SWARM}

\section{A. Position Information in Generic Radio Signals}

In this paper, we place emphasis on exploiting radio signals for swarm navigation. Before diving into the assembling of a particular swarm navigation system, we first introduce a generic radio signal model and present the position information embedded in the signal model. Assume a radio signal $s_{v}(t)$ is modulated onto a carrier $e^{j \omega t}$ with carrier frequency $f$ and $\omega=2 \pi f$. This signal is transmitted from an antenna at node $v$, propagates through the line-of-sight (LOS) path, denoted with the index ' 0 ', and $L$ non-line-of-sight (NLOS) paths and reaches an antenna at another node $u$ as

$$
r_{u v}(t)=\sum_{l=0}^{L} \alpha_{u v, l} s_{v}\left(t-(\overbrace{d_{u v, l} / c-\delta_{u v}}^{\tau_{u v}, l})\right)+\epsilon(t),
$$

with the path length $d_{u v, l}$, the relative clock offset $\delta_{u v}$, the speed of light $c$, the path delay $\tau_{u v, l}$ and the circularlysymmetric complex valued Gaussian noise $\epsilon(t)$. The path amplitude $\alpha_{u v, l}$ can be further expanded with real valued magnitude $A_{u v, l}$ and phase $\Phi_{u v, l}$ as

$$
\alpha_{u v, l}=A_{u v, l} e^{-j(\overbrace{\omega d_{u v, l} / c-\phi_{u v}}^{\Phi_{u v, l}},},
$$

where $\phi_{u v}$ denotes the relative phase offset. In principle the position information can also be extracted by the path magnitude $A_{u v, l}$ and the NLOS components. However, most radio navigation systems exploit the phase and signal delay of the LOS path, i.e. $d_{u v, 0}=\left\|\mathbf{p}_{u}-\mathbf{p}_{v}\right\|$, due to their accuracy and observation simplicity. The notation $\|\cdot\|$ denotes the Euclidean norm of a vector. The signal delay $\tau_{u v, 0}$ can be directly utilized to estimate the distance between node $u$ and $v$, once the clock offset is compensated, for example by two way ranging. Extracting distance directly from the phase $\Phi_{u v, 0}$ is difficult due to the integer ambiguity. Therefore, phase difference is often exploited for example in direction of arrival (DoA) estimation. In this case a multi-port antenna system with coherent multi-channel reception, like a phased array or a multi-mode antenna (MMA), is required. It is worth to mention that once successfully localized, a swarm can be considered as a distributed phased array, which can collaboratively sense the sources of low frequency radio signals, in applications like LOFAR and return-to-base, see Sec. II-D.

\section{B. Self-Organized Channel Access}

For a swarm navigation system, it is essential to design a self-organized channel access scheme, which guarantees accurate localization with a high update rate and low-latency data exchange [15]. The widely applied opportunistic access schemes like the carrier-sense multiple access with collision avoidance (CSMA-CA) is not suitable for a swarm. The increasing back-off time in CSMA-CA may lead to an unbounded latency, which is unacceptable for both localization and data exchange. Self-organized time-division multiple access (SOTDMA) is preferable for a swarm navigation system 


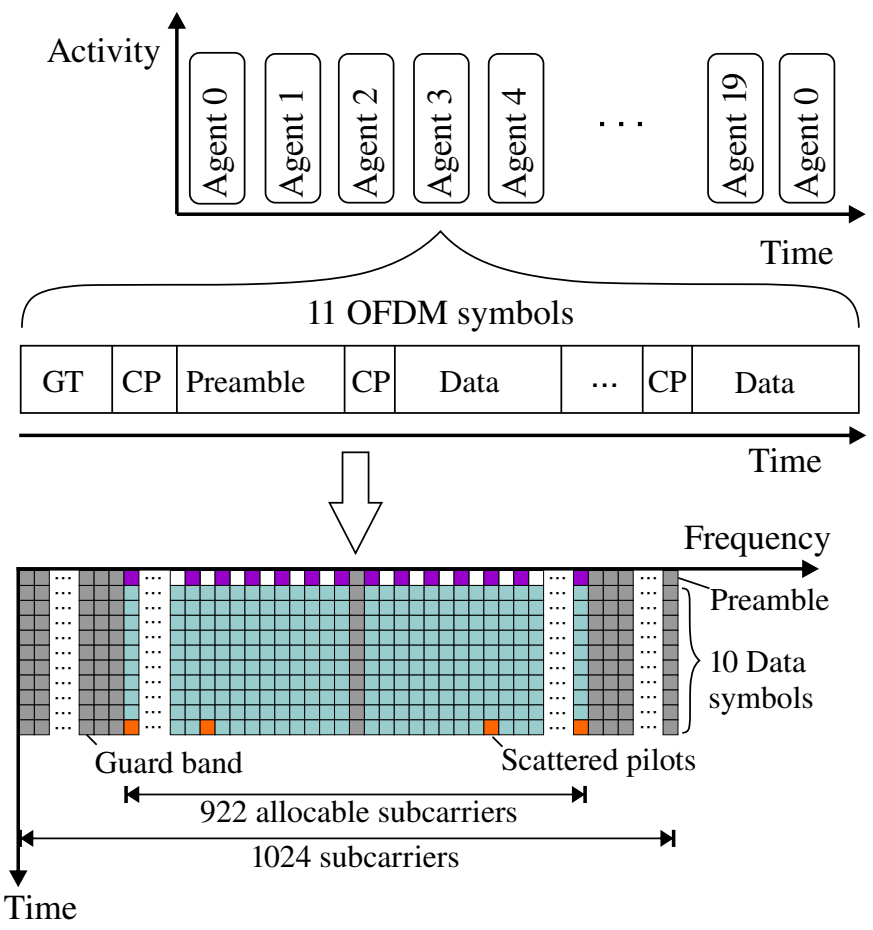

Figure 2: TDMA and OFDM signal structure with GT as TDMA slot guard time and $\mathrm{CP}$ as cyclic prefix.

due to its interference-free exclusive bandwidth (BW) occupation, which is advantageous for localization. In addition, once a time-division multiple access (TDMA) structure has been setup, the latency is upper bounded by the TDMA frame length. Conventionally, an SOTDMA structure, like used in the automatic identification system (AIS) in maritime communications, is setup with a fixed slot length and frame length. Therefore, it is suitable for a swarm with a relatively stable topology and long duration signals like the orthogonal frequency-division multiplexing (OFDM) signal in $3^{\text {rd }}$ generation partnership project (3GPP)-long-term evolution (LTE). An SOTDMA frame structure can alternatively emerge with the concept of pulse coupled oscillator (PCO) [16]. In the PCObased SOTDMA, the TDMA slot length is inherently adaptive, suitable for changing topology and splitting and rejoining of swarms. It is suitable for impulse radio used by ultra-wide band (UWB), but sub-optimal for signals with long duration. In our current swarm navigation system, OFDM signals similar as in LTE are designed for inter-agent communications and ranging. Therefore, we applied an SOTDMA similar to the conventional one to enable self-organized channel access.

\section{OFDM Signal for Swarm Synchronization and Ranging}

Our overall design is driven by the following aspects: Spatio-temporal characteristics of sensed processes, the expected radio channel on a lunar or Martian surface, mobility of agents, properties of available light-weight software-defined radios (SDRs), robustness against agent failures, and a simplified interfacing for its usage in the robotics domain.

In a first step we designed the MAC driven by the distributed algorithms for self-localization and sensing, the mobility of the swarm in conjunction with spatio-temporal correlation of process parameters, and the used low-cost clocks. Distributed algorithms often require iterations among agents to converge, and hence, the radio channel must be accessed frequently. We designed the MAC for a network update rate of $100 \mathrm{~Hz}$ for 20 agents, resulting in slot lengths of $500 \mu \mathrm{s}$. Figure 2 shows the TDMA framing. Each agent has interference-free access on the radio channel for broadcasting, and self-synchronizes its own transmission slot relative to TDMA slots of its neighbors. With this approach, no central master providing slot-boundaries is required, and a single-point of failure is removed from the network.

In a second step we designed the PHY based on OFDM, which is driven by the expected radio channel characteristics, maximum carrier frequency, and swarm mobility. The maximum carrier frequency is $5.9 \mathrm{GHz}$ and the expected maximum relative speed among agents is $100 \mathrm{~km} \mathrm{~h}^{-1}$. Radio propagation models based on channel-sounding measurements for carrier frequencies above $1 \mathrm{GHz}$ are not available for lunar and Martian surfaces. Therefore, we take reference in vehicleto-vehicle, air-to-air, and air-to-ground channel models in the $5 \mathrm{GHz}$ frequency band in hilly terrain environments and define a maximum expected radio channel excess delay of $2 \mu$ s. The final OFDM system parameters yield 1024 subcarriers for a system bandwidth of $25 \mathrm{MHz}, 2 \mu$ s of cyclic-prefix length, 11 OFDM symbols per TDMA slot, and a TDMA slot guard time of $6.76 \mu$ s to take a maximum distance of $2 \mathrm{~km}$ between two agents into account. Only $90 \%$ of the system bandwidth is allocated resulting in guard bands at the spectrum's edge to take filter responses of radio frequency (RF) signal chains into account. The number of allocable subcarriers is therefore 922 .

The first OFDM symbol is an agent-specific preamble based on Zadoff-Chu codes and is used for frame-synchronization, agent-identification, channel estimation, precise ranging and clock tracking. Preambles are designed for differential correlation resulting in low processing effort to maintain the TDMA frame structure. The following 10 OFDM symbols can be freely allocated for data transmission, where a state-of-the-art (s.o.t.a.) 3GPP-LTE or $5^{\text {th }}$ generation mobile networks (5G) coding and modulation scheme can be used. For ranging and localization we dedicate up to 3 OFDM data symbols with a very strong forward error correction (FEC). Automatic repeat request (ARQ) schemes are not applicable for ranging and localization data, as the information broadcasted by one agent in one TDMA slot is already outdated in the next TDMA frame. Hence, data related to ranging and localization must be decoded error-free under the worst reception conditions. However, ARQ schemes can be used for time-insensitive data transmission in the remaining OFDM symbols. The last OFDM symbol contains scattered pilots used for 2D-filtering of the channel transfer function for all data symbols.

\section{Sensing Low-Frequency Radio Signal}

We have identified two use-cases for sensing a lowfrequency radio signal within a carrier frequency range of $1 \mathrm{MHz}$ to $100 \mathrm{MHz}$ with a robotic swarm: a LOFAR and 
return-to-base navigation, as illustrated in Figure 1. Both usescases demand a high self-localization accuracy in the order of decimeters, a high time-synchronization accuracy in the order of nanoseconds. A LOFAR is a distributed phase-coherent antenna array for radio astronomy. Such an array shall be placed on the lunar far-side to observe the radio-sky without man-made interference and will consist of dozens to hundreds of distributed antenna elements. In return-to-base navigation a robotic swarm shall navigate back to a lander through observing a low-frequency radio beacon. Swarm control is key for this use case: the robotic swarm must span a distributed antenna array with optimal relative positions to jointly achieve highly accurate self-localization and radio beacon source localization.

\section{State ESTIMATION IN A SWARM}

\section{A. Swarm Localization}

A brief survey on network localization is provided in [17], which can be generalized to a wide range of specific applications including swarm self-localization. In particular for swarm localization, we are interested in decentralized localization algorithms suitable for large-scale dense networks with high reliability and low complexity. There are two major challenges in designing decentralized swarm localization algorithms: (1) incorporating neighbors' position uncertainty while remaining low complexity, and (2) minimizing the stochastic propagation effects, e.g. multipath and NLOS propagation. We proposed an algorithm dubbed direct particle filtering for decentralized network localization (DiPNet) in [18], which explicitly conquers these challenges. DiPNet adapts the sum-product algorithm over a wireless network (SPAWN) algorithm introduced in [19] to account for position uncertainty of neighboring nodes, with further reduced complexity. In addition, it exploits the concept of direct position estimation (DPE), directly considering the received signals as measurements, instead of taking the range measurements as in traditional two step localization algorithms. This approach exploits the advantage of a swarm, that is a single measurement failure can be collaboratively corrected by the highly redundant measurements. The propagation effects on DiPNet become insignificant for dense networks, due to the multi-link collective PHY layer signal processing. Consequently, DiPNet achieves a near-optimal performance with low complexity, which is particularly attractive for realtime dense-network localization.

Next, we provide a compact description of DiPNet and refer the interested reader to [18] for more details. Assume a swarm $\mathcal{A}$ with $|\mathcal{A}|$ agents at positions $\mathbf{p}=\operatorname{vec}\left\{\mathbf{p}_{u}: u=1, \cdots,|\mathcal{A}|\right\}$. An optimal decentralized Bayesian localization at agent $u$ estimates its own position using the marginalized a posteriori probability density function (pdf),

$$
p\left(\mathbf{p}_{u} \mid \mathbf{z}\right) \propto p\left(\mathbf{p}_{u}\right) \int p\left(\mathbf{p}_{/ u} \mid \mathbf{p}_{u}\right) \prod_{v, w=1}^{|\mathcal{A}|} p\left(\mathbf{z}_{v w} \mid \mathbf{p}_{v}, \mathbf{p}_{w}\right) \mathrm{d} \mathbf{p} / u
$$

where $\mathbf{p} / u$ is the positions of agents other than $u$ and $\mathbf{z}_{v w}$ is the measurement between agents $v$ and $w$. Due to cooperation among agents, a $2(|\mathcal{A}|-1)$ dimensional integral is needed for an exact decentralized Bayesian estimator of $\mathbf{p}_{u}$, which makes it impracticable. A popular approach to reduce the complexity of marginalization is belief propagation (BP), for example the SPAWN algorithm [19]. In SPAWN, an agent $u$ approximates its marginalized a posteriori pdf $p\left(\mathbf{p}_{u} \mid \mathbf{z}\right)$ by the belief $b_{u}^{(K)}$, which is updated by exchanging belief with neighbors $\mathcal{A}_{u}$ for $K$ iterations

$$
b_{u}^{(k)}=b_{u}^{(0)} \prod_{v \in \mathcal{A}_{u}} \int b_{v}^{(k-1)} p\left(\mathbf{z}_{u v} \mid \mathbf{p}_{u}, \mathbf{p}_{v}\right) \mathrm{d} \mathbf{p}_{v} \quad k=1, \cdots, K .
$$

The SPAWN reduces the complexity to $\left|\mathcal{A}_{u}\right|$ integrals with four dimensions for each agent at each iteration. Depending on the distribution model, the marginalization in (4) can be realized by Monte Carlo integration as in non-parametric belief propagation (NBP) [20], by numerical integration for example in cubature belief propagation (CBP) [21], or analytically with parametric belief propagation (PBP) for special distributions [22]. For the highly non-Gaussian and non-linear models involved in localization, NBP is often applied [23]. The proposed DiPNet is a variant of $\mathrm{BP}$, where non-parametric belief is updated locally and only the first two moments of the belief $b_{u}^{(k)}$ are broadcasted to the neighbors. In order to further reduce the complexity, we propose a belief update based on the Fisher information (FI) theory. Hence, the mean square error (MSE) matrix $C_{\hat{\mathbf{p}}}$ of the position estimate $\hat{\mathbf{p}}$ can be assessed by the FI in snapshot case, and the Bayesian information (BI) in tracking case, i.e.

$$
C_{\hat{\mathbf{p}}} \succcurlyeq \mathbf{I}_{\mathbf{p}}^{-1}
$$

We denote either the position $\mathrm{FI}$ or $\mathrm{BI}$ by $\mathbf{I}_{\mathbf{p}}$ to include both snapshot and tracking cases. With the help of FI, the so called equivalent measurement likelihood (EL), denoted as $\tilde{p}^{(k-1)}\left(\mathbf{z}_{u v} \mid \mathbf{p}_{u}, \mathbf{p}_{v}\right)$, can be found. The belief update can then be simplified as

$$
b_{u}^{(k)}=b_{u}^{(0)} \prod_{v \in \mathcal{A}_{u}} \tilde{p}^{(k-1)}\left(\mathbf{z}_{u v} \mid \mathbf{p}_{u}, \mathbf{p}_{v}\right) \quad k=1, \cdots, K .
$$

The concept of EL can be applied to two-step localization [24] or to DPE as in DiPNet.

\section{B. Swarm Attitude Estimation}

The attitude of a robot can be represented by the three Euler angles roll, pitch and yaw. Knowing them is essential for control, e.g. to drive in a certain direction, but also for placement of sensors, manipulation etc. While roll and pitch can be determined from the gravity vector, which is measured by accelerometers, estimating yaw is more challenging. Starting from a known yaw, the relative changes could be obtained by integrating the turnrate measured by a gyroscope. However, due to drifting sensor biases, this leads to a quickly growing error over time. On earth, the magnetic field could be used to determine the yaw. For extraterrestrial exploration, this is in 
general not an option, as e.g. Moon or Mars do not have a dipole magnetic field like the earth.

Instead, in our approach we estimate the yaw based on the DoA of received radio signals, i.e. azimuth $\gamma_{u v, l}$ and elevation $\theta_{u v, l}$ of the impinging signal. Classically, phased antenna arrays are used for that purpose [25]. A phased array typically consists of multiple identical antenna elements in a certain arrangement, often with uniform spacing. Depending on the direction, an impinging signal arrives with slightly different phase at the different antenna elements. For lowpower devices, another approach is to use multiple directive antennas, and compare the received signal strength indicator (RSSI), i.e. the signal power [26], [27]. Recently, MMAs have been proposed as a new approach for DoA estimation [28]. They are designed based on the theory of characteristic modes, where each mode is excited independently. For an MMA, the direction information is contained in both power and phase of the signals. First measurements results with an MMA prototype mounted on rover are presented in [29]. All three antenna types can be described by their antenna response

$$
a_{m}(\gamma)=\sqrt{g_{m}\left(\gamma_{u v, l}, \theta_{u v, l}\right)} e^{\mathrm{j} \Xi_{m}\left(\gamma_{u v, l}, \theta_{u v, l}\right)},
$$

which contains amplitude variations $\sqrt{g_{m}\left(\gamma_{u v, l}, \theta_{u v, l}\right)}$ and phase variations $\Xi_{m}\left(\gamma_{u v, l} \theta_{u v, l}\right)$ for all antenna ports $m=$ $1, \ldots, M$. The signal model (1) can then be extended to $M$ channels $\mathbf{r}_{u v}(t)=\left[r_{u v}^{1}(t), \ldots, r_{u v}^{M}(t)\right]^{T}$, which yields

$$
\mathbf{r}_{u v}(t)=\sum_{l=0}^{L} \mathbf{a}\left(\gamma_{u v, l}, \theta_{u v, l}\right) \alpha_{u v, l} s_{v}\left(t-\tau_{u v, l} / c\right)+\boldsymbol{\epsilon}_{u v}(t)
$$

Assuming for simplicity all agents are located in a flat plane, i.e. $\theta_{u v}=0^{\circ}$, the azimuth DoA is

$$
\gamma_{u v}=\arctan 2\left(y_{v}-y_{u}, x_{v}-x_{u}\right)-\psi_{u},
$$

where $\psi_{u}$ is the yaw of agent $u$. Equation (9) shows that position estimates of the agents in a common coordinate system are required, in order to convert DoA into attitude information. While it may seem that the DoA is only useful for attitude estimation, in [30] it was shown that, depending on how far the agents are apart, it could also contain valuable position information.

\section{INFORMATION SEEKING SWARM CONTROL}

In autonomous swarm control, the swarm decides by itself a control command $\mathbf{b}$ according to certain mission objectives. The position of the swarm is then steered by the control command from $\mathbf{p}$ to $\mathbf{p}^{(+)}$, i.e. $\mathbf{p}^{(+)}=f(\mathbf{p}, \mathbf{b})$. For traditional navigation systems, the control objectives are externally defined, optionally tolerating the position uncertainty. Contrarily, in our autonomous swarm navigation system the position uncertainty is a controllable feature. The swarm is aware of not only the position uncertainty but also the causality between position and its uncertainty. This causality is inferred by the gradient of the weighted position MSE matrix $\Lambda C_{\hat{\mathbf{p}}}$, with respect to (w.r.t.) the control command $\mathbf{b}$. The weighing matrix $\Lambda$ can be chosen flexibly according to applications.
Similarly as in VI-A, the position MSE matrix is evaluated with FI or BI. With the causality awareness, the swarm can actively optimize its position minimizing the uncertainty, which is referred to as information seeking swarm control. The information seeking can be formulated as a cost function $\Lambda \mathbf{I}_{\mathbf{p}}^{(+)^{-1}}$, which is minimized with best effort. This type of information seeking is preferable in, e.g., low frequency source localization, where the source is considered as a special node in the extended swarm network. Alternatively, the information seeking can be expressed as constraints, $\mathbf{E}_{\max }-\Lambda_{\mathrm{c}} \mathbf{I}_{\mathbf{p}}^{(+)^{-1}} \succcurlyeq 0$, where $\mathbf{E}_{\max }$ is the maximally tolerated position error. This type of information seeking is suitable for, for example, limiting the position error of the agents. In addition to the information seeking, other mission cost functions $\mathbf{f}(\mathbf{b})$, like return-to-mission base, or constraints $\mathbf{h}(\mathbf{b}) \geq \mathbf{0}$, like collision avoidance, can be considered. The overall control objective of the swarm can be expressed as

$$
\begin{array}{ll} 
& \underset{\mathbf{b}}{\operatorname{minimize}}\left\{\operatorname{diag}\left\{\Lambda \mathbf{I}_{\mathbf{p}}^{(+)^{-1}}\right\}, \mathbf{f}(\mathbf{b})\right\} \\
\text { s.t. } & \mathbf{E}_{\max }-\Lambda_{\mathrm{c}} \mathbf{I}_{\mathbf{p}}^{(+)}{ }^{-1} \succcurlyeq \mathbf{0} \\
& \mathbf{h}(\mathbf{b}) \geq \mathbf{0} .
\end{array}
$$

In [9] we have proposed a swarm control strategy based on projected steepest gradient descent (PSGD). The gradient of every objective function w.r.t. the control command is analytically derived, which enables low complexity swarm control.

\section{EXPERIMENTAL PlatForms}

Table I shows the evolution of our swarm communication, navigation, and timing system. The very first generation 1.0 has been based on a custom field-programmable gate array (FPGA) design for time-stamped transmission and reception with frequency-duplex, and amplify-and-forward relaying. A centrally scheduled TDMA with unicast based round trip time (RTT) ranging has been used to obtain very short reply times for RTT.

Our current experimental platforms with generation 2.0 are based on an SDR architecture comprising various types of universal software radio peripherals (USRPs) and the custom PHY and MAC from Sec. II-C in the open-source framework Gnuradio. This approach enables faster prototyping from research to evaluation in experiments, and we can scale the number of agents in a network with lower cost. Figure 3 shows an overview and signal processing flow-graph. Our design enables the usage of heterogeneous hardware, e.g., a single-antenna USRP for self-localization with ranging only, and a multi-channel phase-coherent USRP for additional DoA estimation. A first processing step in the receiver is the OFDM frame synchronization, carrier frequency offset (CFO) compensation, and agent identification. Next, we estimate the DoA and time of arrival (ToA) and in parallel demodulate and decode the OFDM data symbols. Based on ToA estimates and transmit time stamps encoded in the data symbols we track the clock of individual agents with Kalman filters (KFs) and 
Table I: Evolution of our swarm navigation testbed.

\begin{tabular}{llllc}
\hline Gen. & Hardware & Protocol & Metrics & Data \\
\hline 1.0 & Custom FPGA & $\begin{array}{l}\text { TDMA, } \\
\text { unicast }\end{array}$ & Range & no \\
\hline 2.0 & $\begin{array}{l}\text { Single-channel } \\
\text { SDR }\end{array}$ & $\begin{array}{l}\text { SOTDMA, } \\
\text { broadcast }\end{array}$ & Range & yes \\
\hline 2.1 & $\begin{array}{l}\text { Single/multi- } \\
\text { channel SDR }\end{array}$ & $\begin{array}{l}\text { SOTDMA, } \\
\text { broadcast }\end{array}$ & Range, DoA & yes \\
\hline
\end{tabular}

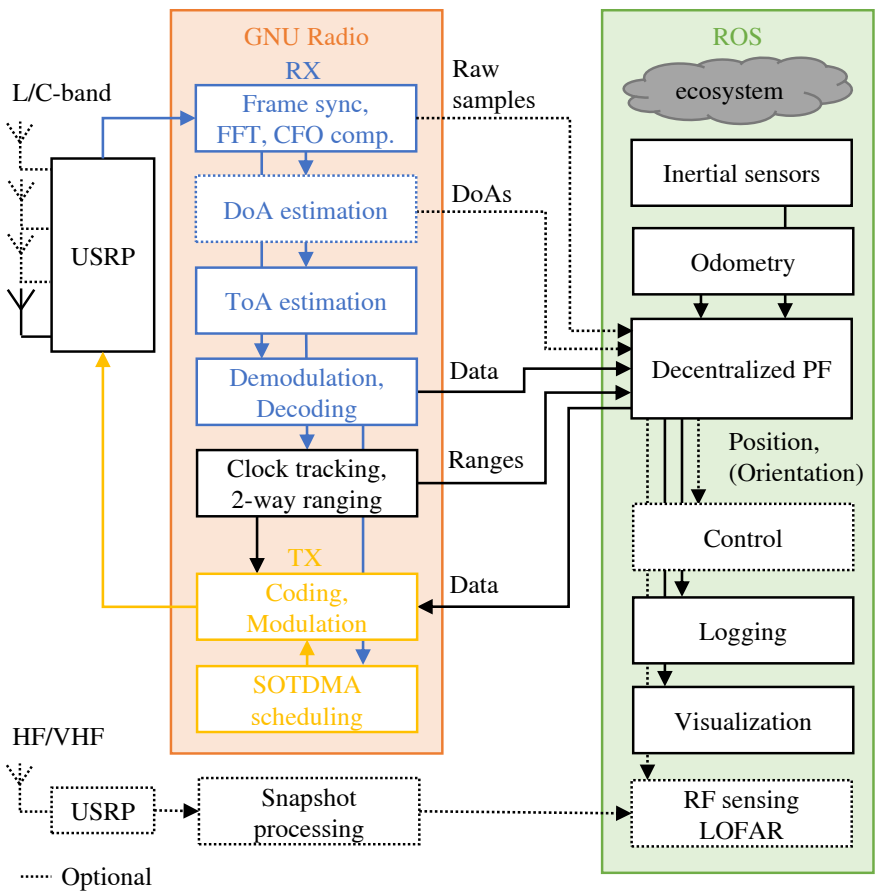

Figure 3: Overview and flow-graph of the swarm communication, navigation, and timing system.

calculate the RTT for distance estimation. On the transmit side, we implemented the necessary coding and modulation, and the MAC for SOTDMA scheduling, which is closely coupled with the PHY.

A challenge besides the design of the SDR architecture, PHY and MAC has been the interfacing into the other swarm subsystems, and hence, into the robotics domain. We utilize the robot operating system (ROS), which is a commonly used middleware in robotics to tackle this challenge. A distributed particle filter (DPF) is realized in ROS with interfaces from and to Gnuradio, e.g., data, ranges, and raw samples. This realization in ROS enables real-time self-localization exploiting ranging information or directly the radio waveform from the SDR, and other robot's sensors available in the ROS ecosystem. Additional sensors including for example inertial sensors and odometry, and estimated agent positions can be used in the ROS ecosystem by other entities. The robot's local control can be accessed, such that information seeking with swarm control can be realized. Sensing low-frequency radio signals is realized with additional USRP interfacing to the ROS ecosystem.
Table II: Our experimental activities.

\begin{tabular}{llll}
\hline Year & Gen. & Environment & New features \\
\hline 2015 & 1.0 & Grass field & DiPNet \\
\hline 2018 & 2.0 & Gravel pit & Real-time DPF \\
\hline 2018 & 2.0 & IAC (indoor) & Larger BW, payload box \\
\hline 2019 & 2.0 & Mt. Etna (volcano) & Scouting, first tests \\
\hline 2020 & 2.1 & Grass field & DoA, ROS \\
\hline 2021 & 2.1 & Mt. Etna (volcano) & LOFAR \\
\hline
\end{tabular}

\section{Selected Results}

Throughout the the evolution of the swarm navigation system, we have conducted numerous measurements and demonstrations. The most important milestones are summarized in Table II. In 2015, we made measurements with the first version of the system, where DiPNet was evaluated. In 2018, as a final project demonstration, six rovers navigated in a gravel pit for gas source exploration. A real-time version of the DPF was implemented for that purpose. In the same year, we demonstrated the system indoors at the International Astronautical Congress (IAC) in Bremen, Germany. The BW was increased and the hardware was integrated into space analogue carbon fibre payload boxes, which can be manipulated by a robot [31]. In 2019 a scout mission took place on the volcano Mt. Etna in Sicily, Italy. In 2020, we integrated a multichannel SDR and an MMA into our experimental platform (generation 2.1), which enables real-time attitude estimation. A LOFAR demonstration as part of a space-analog mission [31] was scheduled in 2020 , but shifted to 2021 due to the COVID-19 pandemic.

\section{A. DiPNet Performance on Grass Field}

We conducted an outdoor experiment with six swarm navigation testbeds (generation 1.0) on a grass field in 2015 [18]. Five stationary testbeds were placed in a pentagonal formation and the sixth testbed was mounted on a remotely controlled rover. The received OFDM symbols of 9900 unicast two-way measurements from 330 snapshots were collected. The rover was driving smoothly with a moderate velocity until snapshot 250 , where maximum velocity and rapid turns started. Three s.o.t.a. DPFs were implemented as comparison to our proposed DiPNet, namely a two-step algorithm with NBP marginalization, a two-step algorithm with EL and a DPE with NBP marginalization. The number of particles in each DPF has been adapted, so that the overall complexity remained consistent. The position root mean square errors (RMSEs) comparison over snapshots are shown in Figure 4. The ranging links were distorted with the multipath components (MPCs) from surrounding metallic structures. Additionally, low signal to noise ratio (SNR) is observed due to ground reflection. In Figure 4, the gray markers at the bottom indicate the snapshots where the mobile testbed received signals from less than three stationary testbeds. The shaded area on the right indicates the 


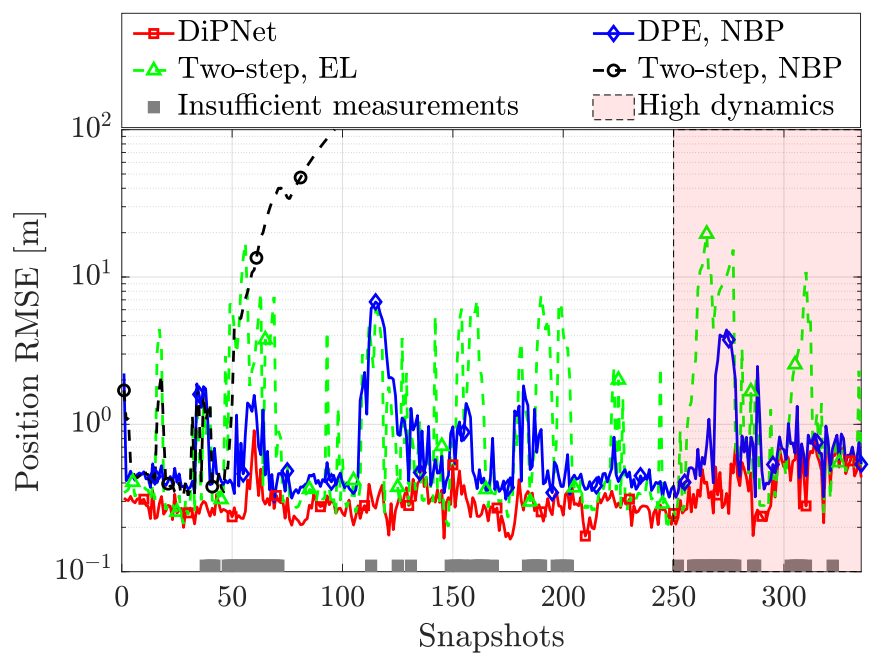

Figure 4: Experimental performance of swarm localization over snapshots: Position RMSE comparison of DiPNet, two-step EL,twostep NBP and DPE-based NBP.

snapshots with a high rover dynamics. DiPNet outperforms all three other algorithms and achieves sub-meter level position accuracy. Higher rover dynamics slightly reduces the DiPNet accuracy due to higher uncertainty in the state transition.

\section{B. Swarm Navigation in a Gas Exploration Mission}

As another milestone of our swarm navigation system (generation 2.0), we have demonstrated the DPF performing in real-time in a gas exploration mission at a gravel pit in 2018. The augmented reality (AR) picture in Figure 5 shows the experimental environment. Three stationary testbeds were located at the corners of the experimental area as anchors with known positions. Five mobile testbeds, the rovers, moved automatically seeking for two emulated sources of gas dispersion [32]. The rovers positions were estimated by the DPF in realtime. The particles are illustrated with blue dots and the point estimates with the red squares. It can be seen that a sub-meter to meter level position estimation accuracy is achieved. In Figure 6 , the ranging performance versus the true distances between all agent pairs are plotted. Accurate range estimates can be obtained with our testbeds, except some outliers due to multipath propagation.

\section{DoA Estimation with MMA}

The latest measurement campagin has been conducted in 2020 on grass field, see Figure 7. Four rovers and three static anchor boxes were deployed. One of the rovers was equipped with an MMA and USRP N310 in a setup with four coherent channels, for details please see [29]. For an MMA, both amplitude and phase carry DoA information. The four receiver channels have been properly calibrated beforehand, in order to compensate phase and magnitude differences. During the measurement, DoA estimation was performed in realtime. The rover was driving for about $10 \mathrm{~min}$, making several turns among and around the anchors. A ground-truth reference for the DoA estimation was provided by a commercial two

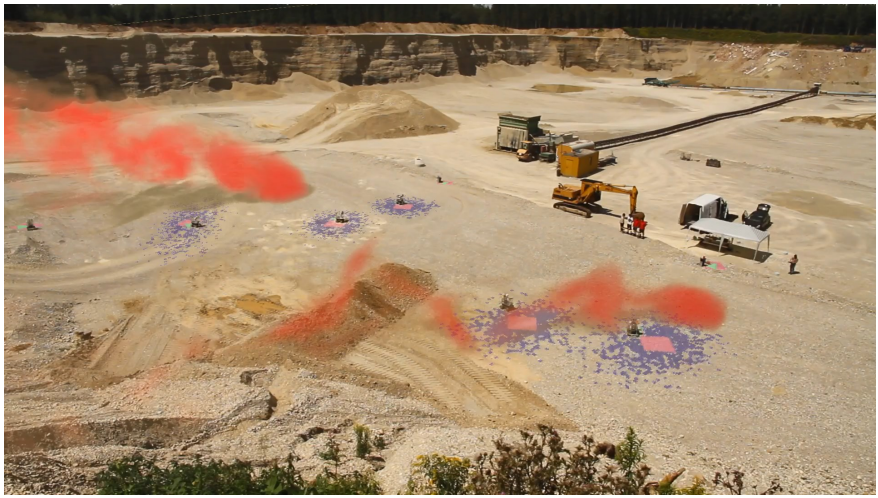

Figure 5: Real-time demonstration of swarm navigation together with a gas exploration mission in a gravel pit in 2018 .

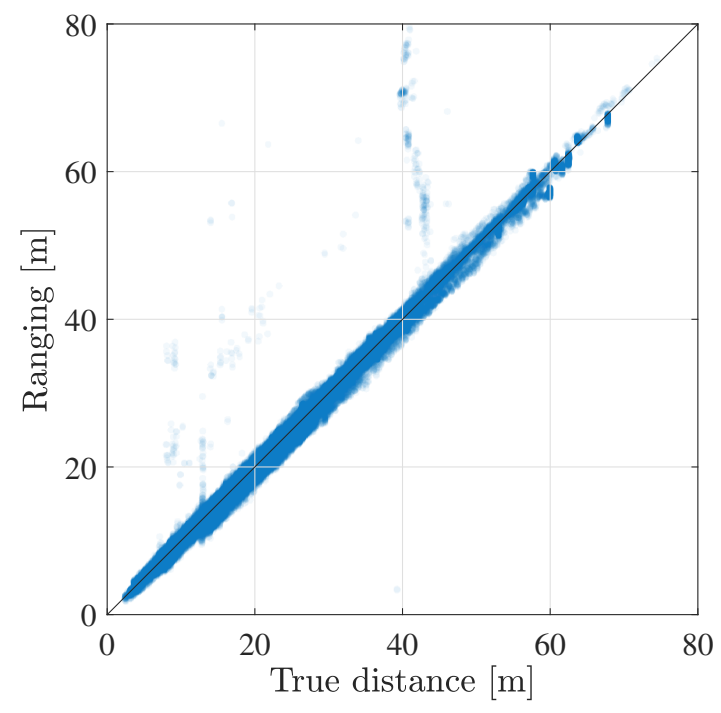

Figure 6: Ranging performance versus true link distances.

antenna real-time kinematic (RTK) system, which internally fuses global navigation satellite system (GNSS) and inertial measurements. Figure 8 shows the estimated DoAs versus the true DoAs w.r.t. the three anchors. For all three links, the estimated and the true DoAs match well, resulting in an RMSE of $3^{\circ} \sim 4^{\circ}$.

\section{Information Seeking Swarm Control}

The information seeking swarm control is a work-inprogress building block, which has been verified with simulations and is not yet integrated in our swarm navigation testbed. We demonstrate here a swarm return-to-base application by simulation. After exploring an area of interest, the swarm intends to return to its mission base. We consider a more challenge case, where only a single low frequency radio source is located at the mission base.

The swarm optimizes its formation to improve the localization performances of itself and the radio source, in order to navigate itself back. 34 agents are considered in the network. Positions of agents and the radio source are exploited as the cost functions in (10). Meantime base approaching is set as 


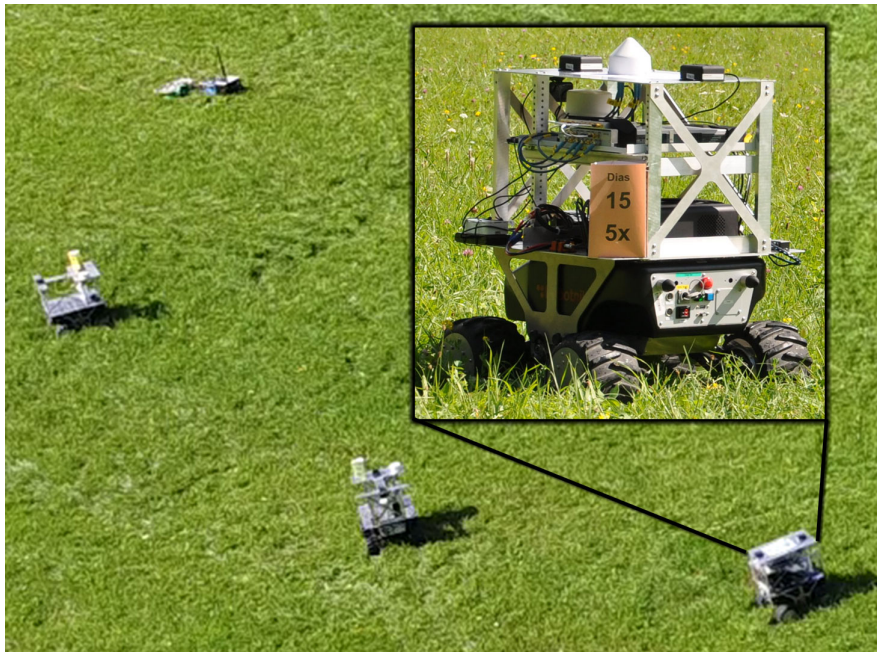

Figure 7: Collecting measurement data with the rovers in 2020 . The highlighted rover features a multi-mode antenna for DoA estimation.

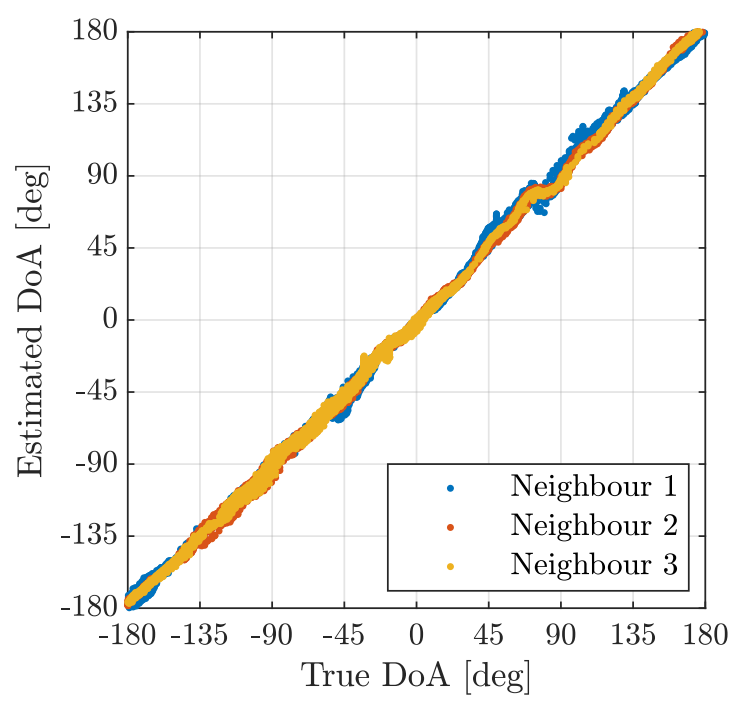

Figure 8: Estimated vs true DoA for signals received from three static neighbours. The DoA is estimated with an MMA mounted on a rover, which is driving for $10 \mathrm{~min}$.

another cost function. The swarm formations at the initial snapshot and after 2000 snapshots are shown in Figure 9. The direction of the radio source is illustrated with the magenta dashed line. Agents are represented as green dots, except the two defining the swarm coordinate system, which are shown as the blue dots. Magenta ellipses indicate the position MSE of agents inferred by FI. The swarm automatically spreads out vertically to the direction of the source, which significantly increases the effective aperture to improve source localization. Meantime, agents remain connected with a regular lattice formation, to perform self-localization. In addition, the two blue reference agents are separated, in order to optimize the swarm coordinate system.

\section{E. Space-Analog Mission on Mt. Etna}

Within the project Autonomous Robotic Networks to Help Modern Societies (ARCHES) we demonstrate technologies
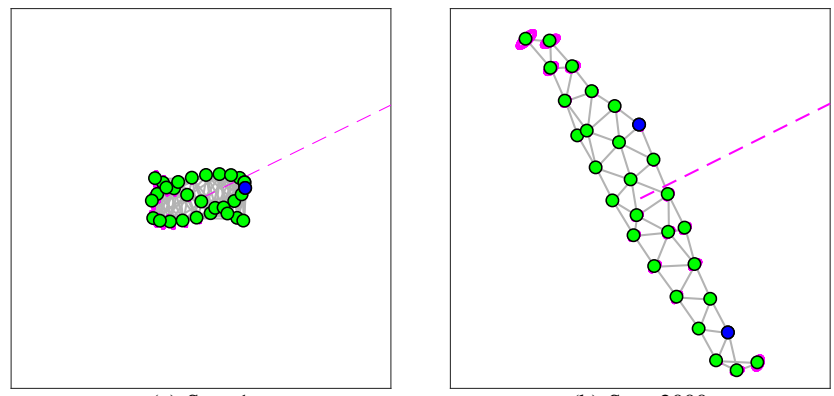

(a) Step 1

(b) Step 2000

Figure 9: FI seeking swarm control for swarm return-to-base application. The formation of the swarm is optimized to improve self- and source localization.

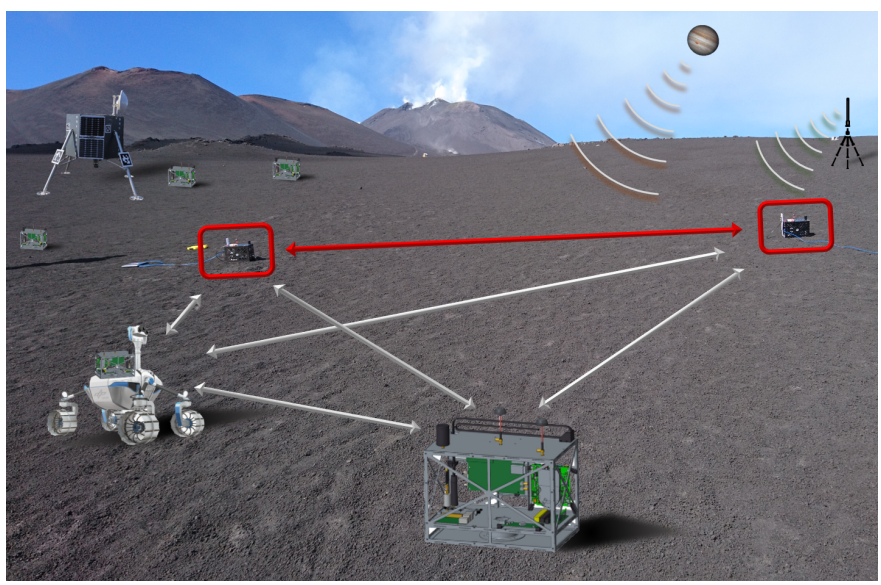

Figure 10: Scouting mission on Mt. Etna in 2019 and the envisioned space-analog LOFAR mission in 2021.

and robotic capabilities in a lunar-analogue environment on Mt. Etna (Sicily, Italy) in 2021 [31]. The demonstration mission consists of three scenarios that scientifically focus on geological research and radio astronomy, which are relevant for future exploration activities. The first two scenarios examine technical and operational aspects of geological in-situ analysis and sample return. The third scenario demonstrates the autonomous installation and maintenance of a LOFAR with heterogeneous robots, which is illustrated in Figure 10. LOFAR payload boxes are deployed by the lightweight rover and precisely synchronized and localized with our swarm navigation system. Low frequency radio signal transmitted either from space or by an artificial transmitter is sensed by this array. As highlighted in red color, the two-way ranging function has been verified with two payload boxes in the scouting mission on Mt. Etna in 2019.

\section{Summary AND CONClusion}

In this paper we have addressed the assembling of a swarm navigation system. We have focused on communication, localization, sensing and control aspects. The proposed swarm navigation system uses OFDM radio signals for both communication between and localization of the swarm system's agents. The radio channel access has been implemented selforganized and distributed among the agents, providing flexibility in terms of the number of agents and preventing single 
points of failure. We have introduced DiPNet as an algorithm which uses a distributed particle filter and estimates the agent's positions directly from the observation of the OFDM radio signals, which are exchanged mutually between the agents. Experimental results for DiPNet show a localization accuracy below $1 \mathrm{~m}$. With that, DiPNet significantly outperforms a traditional two step approach which consists of mutual ranging between the agents and subsequent location determination of the agents using the range estimates between the agents. Orientation estimation based on MMAs has shown an RMSE of $3^{\circ} \sim 4^{\circ}$.

The presented selection of results was obtained using our experimental swarm system platform. Already the current generation of our platform provides evidence that swarm systems are able to navigate with sufficient accuracy in unknown and previously untouched exploration areas like planetary surfaces.

\section{ACKNOWLEDGMENT}

Part of the presented research has been supported by the Helmholtz Association project ARCHES (contract number ZT-0033) and the German Aerospace Center (DLR) project Swarm-Navigation. The authors would like to thank Thomas Wiedemann and Stefano Caizzone for their support.

\section{REFERENCES}

[1] M. Ballerini et al., "Interaction ruling animal collective behavior depends on topological rather than metric distance: Evidence from a field study," Proceedings of the National Academy of Sciences, vol. 105, no. 4, pp. 1232-1237, 2008.

[2] C. W. Reynolds, "Flocks, herds and schools: A distributed behavioral model," in Proc. 14 th Annu. Conf. Computer Graphics and Interactive Techniques, ser. SIGGRAPH '87. New York, NY, USA: ACM, 1987, pp. 25-34.

[3] M. Moussaid, S. Garnier, G. Theraulaz, and D. Helbing, "Collective information processing and pattern formation in swarms, flocks, and crowds," Topics in Cognitive Science, vol. 1, no. 3, pp. 469-497, 2009.

[4] M. G. Hinchey, R. Sterritt, and C. Rouff, "Swarms and swarm intelligence," IEEE Computer, vol. 40, no. 4, pp. 111-113, Apr. 2007.

[5] S. Li, R. Batra, D. Brown, H.-D. Chang, N. Ranganathan, C. Hoberman, D. Rus, and H. Lipson, "Particle robotics based on statistical mechanics of loosely coupled components," Nature, vol. 567, pp. 361-365, Mar. 2019.

[6] M. Bernard, K. Kondak, I. Maza, and A. Ollero, "Autonomous transportation and deployment with aerial robots for search and rescue missions," J. Field Robot., vol. 28, no. 6, pp. 914-931, 2011.

[7] M. Dunbabin and L. Marques, "Robots for environmental monitoring: Significant advancements and applications," IEEE Robot. Autom. Mag., vol. 19 , no. 1, pp. 24-39, Mar. 2012.

[8] A. Seeni, B. Schfer, and G. Hirzinger, "Robot mobility systems for planetary surface exploration - state-of-the-art and future outlook: A literature survey," in Aerospace Technologies Advancements, T. T., Ed. London: InTech, Jan. 2010, pp. 189-208.

[9] S. Zhang, R. Pöhlmann, T. Wiedemann, A. Dammann, H. Wymmeersch, and P. A. Hoeher, "Self-aware swarm navigation in autonomous exploration missions," Proc. IEEE, vol. 108, no. 7, pp. 1168-1195, 2020.

[10] A. L. Christensen, R. O'Grady, and M. Dorigo, "From fireflies to faulttolerant swarms of robots," IEEE Trans. Evol. Comput., vol. 13, no. 4, pp. 754-766, Aug. 2009.

[11] E. Vassev, R. Sterritt, C. Rouff, and M. Hinchey, "Swarm technology at NASA: Building resilient systems," IEEE IT Prof., vol. 14, no. 2, pp. 36-42, Mar. 2012

[12] N. R. Raz and M. Akbarzadeh-T., "Swarm-fuzzy rule-based targeted nano delivery using bioinspired nanomachines," IEEE Trans. Nanobiosci., pp. 1-1, 2019

[13] M. Schwager, B. J. Julian, M. Angermann, and D. Rus, "Eyes in the sky: Decentralized control for the deployment of robotic camera networks," Proc. IEEE, vol. 99, no. 9, pp. 1541-1561, Sep. 2011.
[14] A. Wedler et al., "From single autonomous robots toward cooperative robotic interactions for future planetary exploration missions," in Proc. of the Int. Astronautical Congr., IAC, Bremen, Germany, Oct. 2018.

[15] T. Andre, K. A. Hummel, A. P. Schoellig, E. Yanmaz, M. Asadpour, C. Bettstetter, P. Grippa, H. Hellwagner, S. Sand, and S. Zhang, "Application-driven design of aerial communication networks," IEEE Commun. Mag., vol. 52, no. 5, pp. 129-137, May 2014.

[16] J. Degesys, I. Rose, A. Patel, and R. Nagpal, "DESYNC: SelfOrganizing Desynchronization and TDMA on Wireless Sensor Networks," in 6th International Symposium on Information Processing in Sensor Networks, 2007. IPSN 2007, april 2007, pp. $11-20$.

[17] R. M. Buehrer, H. Wymeersch, and R. M. Vaghefi, "Collaborative sensor network localization: Algorithms and practical issues," Proc. IEEE, vol. 106, no. 6, pp. 1089-1114, Jun. 2018

[18] S. Zhang, E. Staudinger, T. Jost, W. Wang, C. Gentner, A. Dammann, H. Wymeersch, and P. A. Hoeher, "Distributed direct localization suitable for dense networks," IEEE Trans. Aerosp. Electron. Syst., vol. 56, no. 2, pp. 1209-1227, 2020.

[19] H. Wymeersch, J. Lien, and M. Win, "Cooperative localization in wireless networks," Proc. IEEE, vol. 97, no. 2, pp. 427-450, Feb. 2009.

[20] A. T. Ihler, J. W. Fisher, R. L. Moses, and A. S. Willsky, "Nonparametric belief propagation for self-localization of sensor networks," IEEE J. Sel. Areas Commun., vol. 23, no. 4, pp. 809-819, Apr. 2005.

[21] F. Meyer, O. Hlinka, and F. Hlawatsch, "Sigma point belief propagation," IEEE Signal Process. Lett., vol. 21, no. 2, pp. 145-149, Feb. 2014.

[22] J. Lien, U. J. Ferner, W. Srichavengsup, H. Wymeersch, and M. Z. Win, "A comparison of parametric and sample-based message representation in cooperative localization," Int. J. of Navigation and Observation, pp. $1-10,2012$.

[23] F. Meyer, H. Wymeersch, M. Froehle, and F. Hlawatsch, "Distributed estimation with information-seeking control in agent networks," IEEE J. Sel. Areas Commun., vol. 33, no. 11, pp. 2439-2456, Nov. 2015.

[24] S. Zhang, R. Raulefs, A. Dammann, and S. Sand, "System-level performance analysis for Bayesian cooperative positioning: From global to local," in International Conference on Indoor Positioning and Indoor Navigation (IPIN). IEEE, 2013, pp. 1-10.

[25] M. Viberg, "Introduction to array processing," in Array and Statistical Signal Processing, ser. Academic Press Library in Signal Processing, A. M. Zoubir, M. Viberg, R. Chellappa, and S. Theodoridis, Eds. Boston: Elsevier, 2014, vol. 3, ch. 11, pp. 463-502.

[26] A. U. Ahmed, M. T. Islam, and M. Ismail, "Estimating DoA from radio frequency RSSI measurements using multi-element femtocell configuration," IEEE Sensors Journal, vol. 15, no. 4, pp. 2087-2092, Apr. 2015.

[27] M. Hartmann, M. Wohler, M. Schühler, L. Weisgerber, J. Thielecke, and A. Heuberger, "A dual frequency antenna for RSSI-based DOA estimation - From theory to prototype," in Proc. Days Diffraction (DD), Jun. 2016, pp. 182-187.

[28] R. Pöhlmann, S. A. Almasri, S. Zhang, T. Jost, A. Dammann, and P. A. Hoeher, "On the potential of multi-mode antennas for direction-of-arrival estimation," IEEE Transactions on Antennas and Propagation, vol. 67, no. 5, pp. 3374-3386, May 2019.

[29] R. Pöhlmann, G. Pedregosa, S. Caizzone, E. Staudinger, and P. A. Hoeher, "Multi-mode antenna enabled direction-of-arrival estimation for swarm navigation," in Proc. 16th Workshop Positioning, Navigation and Communications (WPNC), Bremen, Germany, Oct. 2019, pp. 1-6.

[30] R. Pöhlmann, S. Zhang, A. Dammann, and P. A. Hoeher, "Fundamental limits for joint relative position and orientation estimation with generic antennas," in Proc. 26th European Signal Processing Conf. (EUSIPCO), Rome, Italy, 2018, pp. 697-701.

[31] M. J. Schuster et al., "The ARCHES space-analogue demonstration mission: Towards heterogeneous teams of autonomous robots for collaborative scientific sampling in planetary exploration," IEEE Robotics and Automation Letters, pp. 1-1, 2020.

[32] T. Wiedemann, D. Shutin, and A. J. Lilienthal, "Model-based gas source localization strategy for a cooperative multi-robot system-a probabilistic approach and experimental validation incorporating physical knowledge and model uncertainties," Rob. Auton. Syst., 2019. 